\title{
Magnetic Quadrupole Formation of Elliptical Sheet Electron Beams for High-Power Microwave Devices
}

\author{
M. A. Basten, J. H. Booske, Senior Member, IEEE, and J. Anderson
}

\begin{abstract}
Sheet electron beams are attractive for high-power microwave sources due to their ability to transport high current, at reduced current density, through thin clearance apertures and in close proximity to walls or RF structures. This paper reports on the theoretical investigation of magnetic quadrupole formation of elliptical sheet electron beams for use in highpower microwave devices. The beam envelope equations for an initially round beam passing through a physical non-symmetric quadrupole pair in the presence of space-charge, finite beam emittance, and under the effects of third-order field components and longitudinal velocity variations are presented. The presence of space-charge compensates for over-focusing in the thin beamdimension and allows for the formation of highly elliptic sheet electron beams. As an example, the results of our study were applied to an existing Pierce gun source with a beam radius of $0.6 \mathrm{~cm}$, beam energy of $10 \mathrm{keV}$ and current density of $2.0 \mathrm{~A} / \mathrm{cm}^{2}$. We find that an elliptical beam with major radius $r_{a}=3.61 \mathrm{~cm}$, minor radius $r_{b}=0.16 \mathrm{~cm}$ and ellipticity $\left(r_{a} / r_{b}\right)$ of 22.5 can be produced with only modest quadrupole gradients of $64 \mathrm{G} / \mathrm{cm}$ and $18 \mathrm{G} / \mathrm{cm}$. Quadrupole formation of elliptical sheet-beams may be particularly suited for experimental research applications since existing round-beam electron guns may be used and changes in beam ellipticity may be made without breaking the vacuum system.
\end{abstract}

\section{INTRODUCTION}

$\mathbf{C}$ ONVENTIONAL sources of microwave radiation commonly utilize round electron beams from Pierce sources. Power output is often increased in these designs by increasing the beam voltage and/or the beam current. Both methods have potential drawbacks. Higher voltage beams require larger and heavier voltage sources which can restrict the mobility of the system. Higher beam current with constant beam size may lead to efficiency degradation through space-charge effects in transport or spatial debunching of the RF-modulated beam. On the other hand, if the current density is maintained but the current is increased (i.e., increasing the beam diameter), the efficiency of the design may be limited by the spatial spread of the beam with respect to the modal field pattern.

Sources utilizing a sheet electron beam are an attractive alternative to round beams for microwave devices. In these designs beam current may be scaled, without an associated increase in current density, by adding current to the wide dimension of the beam. Suitably thin sheet beams coupled to

Manuscript received August 31, 1993. This work was supported in part by the DoD Vacuum Electronics Initiative as managed by AFOSR (Grant No AFOSR-91-0381) and an NSF Presidential Young Investigator Award (ECS9057675).

The authors are with the Electrical and Computer Engineering Department, University of Wisconsin, Madison, WI 53706 USA.

IEEE Log Number 9405689D.
RF structures with rectangular geometry are less susceptible to efficiency loss due to spatial spread in the thin dimension. Both low-voltage slow-wave devices and high-voltage fast-wave configurations suitable for sheet electron beams are currently being investigated [1], [2].

It is well known that sheet beams in axial guide fields are unstable to diocotron modes driven by the $E \times B$ drift velocity shear between the upper and lower edges of the beam ([3], and the references contained therein). However, it has recently been shown that stable transport of sheet beams is possible in periodically cusped magnet (PCM) configurations [4]. In fact, it was shown that the contoured profile of an elliptical sheet beam provides better matching and possibly lower emittance growth than sheet beams with rectangular cross-sections. Prototype PCM focusing configurations are currently being fabricated at UW-Madison for measurement and experimental testing.

With investigations of stable transport and RF interaction with sheet beams underway, the feasibility of producing sheet beams remains to be demonstrated. The design and fabrication of a electron gun that can produce a sheet-beam directly is complicated by the inherent three-dimensional nature of such a source. Computationally-intensive three-dimensional trajectory simulations are necessary, and the lack of azimuthal symmetry may make the fabrication of components more difficult. One alternative is to send a large round beam through a masking aperture in order to generate a sheet, but it raises questions of beam quality through the aperture [2], as well as the desirability of such a technique in long-pulse and $\mathrm{cw}$ tube designs.

An attractive method for the generation of sheet beams is to use the astigmatism of magnetic quadrupoles, and quadrupole pairs, to transform a round beam into an elliptical beam. Elliptical beams will represent a sheet or ribbon beam in the limit of high eccentricity. Additionally, quadrupole formation of elliptical sheet beams has the added research advantage that changes in ellipticity can be made externally to the vacuum envelope by adjusting the quadrupole strengths and separation. While quadrupoles have been investigated at length and are commonly used for beam transport in accelerators, their feasibility for generating elliptical sheet-beams for microwave devices is an unexplored issue.

This paper discusses the theoretical and practical aspects of a quadrupole lens system and is organized in the following manner: First, the single-particle transport of an electron through an ideal quadrupole pair is discussed and it is shown that, without space-charge, it is not possible to 
obtain highly elliptic paraxial output with an initially laminar round beam. Next, the effect of space-charge on the beam envelope is investigated. The coupled beam envelope equations are numerically solved and demonstrate that the presence of space-charge helps to correct the slope of the envelope in the focusing plane so that highly elliptical, paraxial output becomes possible. Finite beam emittance and the higher-order effects of physical quadrupole magnetic fields and longitudinal velocity variations are then investigated. The theory presented here is nonrelativistic, but generalization to a relativistic formulation should be straightforward. Finally, a realistic design case for a highly elliptical output beam using input beam parameters from an existing Pierce gun is presented.

\section{THEORY}

Consider the transport of a single electron of charge $-e$ and mass $m$ through a quadrupole magnetic field as shown in Fig. 1. To first order, assuming hyperbolic magnet pole faces, the magnetic fields are given by [5]

$$
\begin{aligned}
& B_{x}=-\frac{B_{0}}{R_{0}} y \\
& B_{y}=-\frac{B_{0}}{R_{0}} x .
\end{aligned}
$$

Here $B_{0}$ and $R_{0}$ are the quadrupole field and radius at the pole tip. The quantity $B_{0} / R_{0}$ is often referred to as the quadrupole gradient.

Substituting these fields into the non-relativistic equations of motion for the particle gives

$$
\begin{aligned}
& \frac{d^{2} x}{d t^{2}}=\frac{e}{m} v_{z} B_{y}=-\frac{e}{m} v_{z 0} \frac{B_{0}}{R_{0}} x \\
& \frac{d^{2} y}{d t^{2}}=-\frac{e}{m} v_{z} B_{x}=+\frac{e}{m} v_{z 0} \frac{B_{0}}{R_{0}} y
\end{aligned}
$$

where the longitudinal velocity is assumed to be approximately constant. Replacing $\frac{d}{d t} \rightarrow v_{z 0} \frac{d}{d z}$ gives the following ray equations describing the particle motion

$$
\begin{aligned}
& x^{\prime \prime}=\frac{d^{2} x}{d z^{2}}=-\frac{e}{m} \frac{B_{0}}{R_{0}} \frac{1}{v_{z 0}} x=-k_{0}^{2} x \\
& y^{\prime \prime}=\frac{d^{2} y}{d z^{2}}=\frac{e}{m} \frac{B_{0}}{R_{0}} \frac{1}{v_{z 0}} y=k_{0}^{2} y .
\end{aligned}
$$

Here $k_{0}{ }^{2}\left(=\frac{e}{m} \frac{B_{0}}{R_{0}} \frac{1}{v_{z 0}}\right.$ ) is called the quadrupole excitation. Primed quantities denote derivatives with respect to $z$. The solutions to these equations represent converging (in the focusing plane) and diverging (in the defocusing plane) motion of the particle and are given by [6]

$$
\begin{aligned}
x(z) & =x_{0} \cos k_{0} z+x_{0}{ }^{\prime} \sin k_{0} z \\
x^{\prime}(z) & =-k_{0} x_{0} \sin k_{0} z+x_{0}{ }^{\prime} \cos k_{0} z \\
y(z) & =y_{0} \cosh k_{0} z+y_{0}{ }^{\prime} \sinh k_{0} z \\
y^{\prime}(z) & =k_{0} y_{0} \sinh k_{0} z+y_{0}{ }^{\prime} \cosh k_{0} z
\end{aligned}
$$

where $x_{0}, x_{0}{ }^{\prime}, y_{0}, y_{0}{ }^{\prime}$ refer to the position and slope of the incident particle.

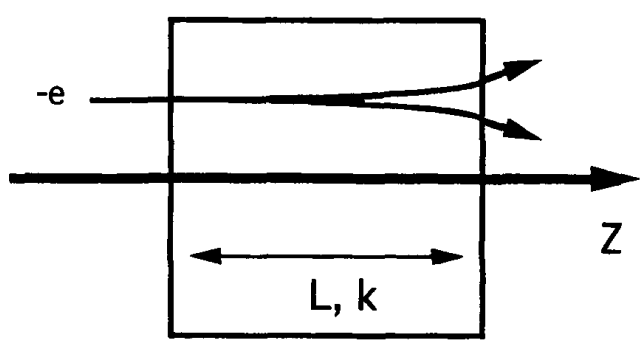

(a)

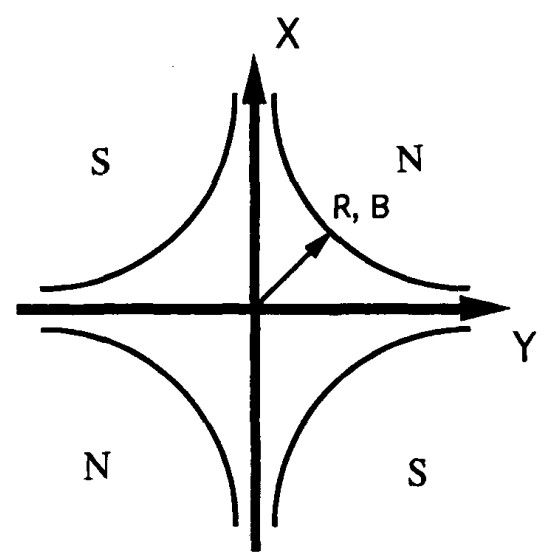

(b)

Fig. 1. Schematic of a quadrupole lens.

Consider the case of an electron moving through two identical quadrupole magnets having the same excitation $\left(k_{1}=\right.$ $k_{2} \equiv k_{0}$ ), length $L$, and separated by a drift distance of length $d$. The second quadrupole is rotated through an azimuthal angle of $\pi / 2$ with respect to the first magnet in order to form a symmetric quadrupole pair. Thus the motion in one plane (the $x-z$ plane) is focusing-drift-defocusing and in the other plane $(y-z)$ the electron's motion is defocusing-drift-focusing. The quadrupole fields are first-order and assumed constant over $L$, and the drift region is assumed to be field-free. If the input trajectory of the electron is assumed to be paraxial (e.g. $x_{0}{ }^{\prime}=0, y_{0}{ }^{\prime}=0$ ) then the slope of the particle as it leaves the second quadrupole is given by

$$
\begin{aligned}
x_{f}^{\prime}= & k_{0} x_{0}\left[\cos k_{0} L \sinh k_{0} L-k_{0} d \sin k_{0} L \sinh k_{0} L\right. \\
& \left.-\sin k_{0} L \cosh k_{0} L\right] \\
y_{f}^{\prime}= & k_{0} y_{0}\left[\cos k_{0} L \sinh k_{0} L-k_{0} d \sin k_{0} L \sinh k_{0} L\right. \\
& \left.-\sin k_{0} L \cosh k_{0} L\right] .
\end{aligned}
$$

If paraxial output from the quadrupole lens system is desired, then the term in brackets in (6) must be zero. Furthermore, we physically require $d \geq 0$, which leads to the condition $\tanh \left(k_{0} L\right) \geq \tan \left(k_{0} L\right)$. This condition is strictly only true when $k_{0} L=0$, but is approximately satisfied in the limit $k_{0} L \rightarrow 0$. This thin-lens limit is only valid for the case where the transverse displacement of the ray through the 
lens system is sufficiently slight such that the force restoring the trajectory to paraxial flow is approximately equal to the original bending force. Thus, highly elliptic paraxial output flow for a matched input beam is not possible in the symmetric quadrupole lens pair without beam space-charge. Although the algebra is more involved and not presented here, it may be more generally demonstrated that, without space-charge, highly elliptic paraxial flow is not possible in either the nonsymmetric pair $\left(k_{1} \neq k_{2}\right)$ or a symmetric quadrupole triplet $\left(k_{1}=k_{3}=k_{2} / \sqrt{2}\right)$.

With the addition of beam space-charge in the model the situation is considerably altered, and highly elliptical, nearparaxial flow is possible. In the focusing plane, the relatively thin dimension of the beam induces relatively large spacecharge electric field forces which act to correct the trajectory in the drift region. Additionally, space-charge pushes out the envelope in the defocused direction. Careful adjustment of the second quadrupole position and gradient allows for highly elliptic paraxial flow in both planes.

After Lapostolle [7], the electric self-field for an elliptical beam with constant charge density $(n e)$ may be approximated by

$$
\begin{aligned}
& E_{x}^{(s)}=-\frac{m}{e} \omega_{p}^{2} \frac{Y}{X+Y} x \\
& E_{y}^{(s)}=-\frac{m}{e} \omega_{p}^{2} \frac{X}{X+Y} y .
\end{aligned}
$$

Here $X$ and $Y$ correspond to the minor and major radii of the elliptical beam cross-section, respectively, and $\omega_{p}(=$ $\left.n e^{2} / \epsilon_{0} m\right)$ is the electron plasma frequency. In the limit of a circular beam $\left(X=Y=R_{b 0}\right)$, we see that (7) give the correct form of the beam self-field. Similarly, in the limit $Y \gg X$, the field component in the $x$ direction approaches that of an infinitely long charged sheet.

In the transformation from circular to elliptical crosssection, the total beam area is not conserved and the charge density is a function of $z$. Assuming that the initially uniformdensity beam remains uniform in the transformation, we may rewrite the plasma frequency as

$$
\omega_{p}^{2}(z)=\omega_{p 0}^{2} A_{b 0} / A_{b}(z)=\omega_{p 0}^{2} \frac{R_{b 0}^{2}}{X Y}
$$

where $A_{b}$ is the beam cross-sectional area and the subscript (o) refers to that parameter at the quadrupole entrance. As we are interested in the beam envelope trajectory, we let $x \rightarrow X$ and $y \rightarrow Y$ and recast (7) as

$$
\begin{aligned}
& E_{x}{ }^{(s)}=-\frac{m}{e} \omega_{p 0}{ }^{2} \frac{R_{b 0}{ }^{2}}{X+Y} \\
& E_{y}{ }^{(s)}=-\frac{m}{e} \omega_{p 0}{ }^{2} \frac{R_{b 0}{ }^{2}}{X+Y}
\end{aligned}
$$

It is appropriate at this point to include an emittance term in the equations of motion to model the case of an finite emittance beam. After Lawson [8], the effect of electron transverse kinetic pressure on the beam envelope may be included as

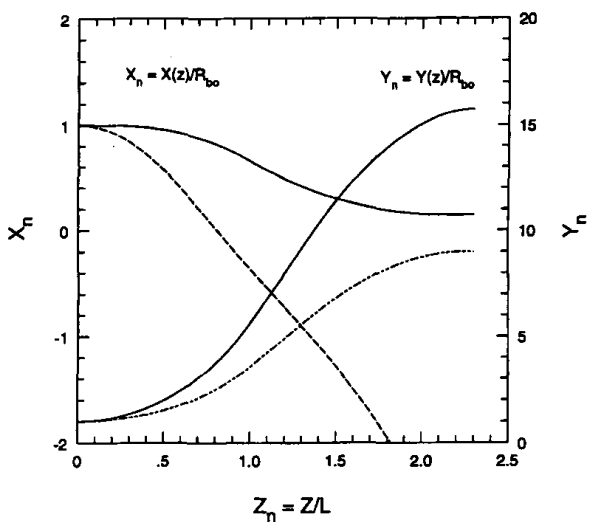

Fig. 2. Beam envelope trajectories through the magnetic quadrupole pair in the presence of space-charge (solid curves) and without space-charge (dashed curves). The envelope trajectory in the $x z$ plane $\left(X_{n}\right)$ is strongly defocused due to space-charge and highly elliptic paraxial flow is possible.

additional defocusing terms of the form

$$
\frac{\epsilon_{n x}^{2}}{X^{3}}, \frac{\epsilon_{n y}^{2}}{Y^{3}}
$$

where $\epsilon_{n x}$ and $\epsilon_{n y}$ are the normalized beam emittances in the $x$ and $y$ directions. The envelope equations for the beam with space-charge and finite emittance now become

$$
\begin{aligned}
X^{\prime \prime} & =-k_{0}{ }^{2} X+\frac{\omega_{p 0}^{2}}{v_{z 0}^{2}} \frac{R_{b 0}^{2}}{X+Y}+\frac{\epsilon_{n x}^{2}}{X^{3}} \\
Y^{\prime \prime} & =k_{0}{ }^{2} Y+\frac{\omega_{p 0}^{2}}{v_{z 0}^{2}} \frac{R_{b 0}^{2}}{X+Y}+\frac{\epsilon_{n y}^{2}}{Y^{3}} .
\end{aligned}
$$

For ease of calculation, we normalize the ray equations with $z_{n}=z / L, X_{n}=X / X_{0}=X / R_{b 0}, Y_{n}=Y / Y_{0}=Y / R_{b 0}$ and $\frac{d}{d z}=\frac{1}{L} \frac{d}{d z_{n}}$. We may now write (11) and (12) as

$$
\begin{aligned}
X_{n}^{\prime \prime}= & -\left(k_{0} L\right)^{2} X_{n}+\left(k_{p} L\right)^{2} \frac{1}{X_{n}+Y_{n}} \\
& +\left(\frac{L}{R_{b 0}}\right)^{2}\left(\frac{\epsilon_{n x}}{R_{b 0}}\right)^{2} \frac{1}{X_{n}^{3}} \\
Y_{n}^{\prime \prime}= & +\left(k_{0} L\right)^{2} Y_{n}+\left(k_{p} L\right)^{2} \frac{1}{X_{n}+Y_{n}} \\
& +\left(\frac{L}{R_{b 0}}\right)^{2}\left(\frac{\epsilon_{n y}}{R_{b 0}}\right)^{2} \frac{1}{Y_{n}^{3}}
\end{aligned}
$$

where primed quantities now denote the derivative with respect to $z_{n}$ and we have defined $k_{p}=2 \pi / \lambda_{p}=\omega_{p 0} / v_{z 0}$. Equations (13) and (14) are coupled through the space-charge term and are valid in the limit $X_{n}^{\prime}, Y_{n}^{\prime} \ll 1$.

To illustrate the effect of space-charge on ellipse formation we numerically solve the above equations for a zero-emittance laminar beam entering an ideal nonsymmetric quadrupole pair. The quadrupoles are separated by a field-free drift region of length $d$. Fig. 2 shows the trajectory of the beam envelope in the focusing and defocusing planes. The beam has an initial current density of $30 \mathrm{~A} / \mathrm{cm}^{2}$ and energy of $10 \mathrm{keV}$. The first and second quadrupoles have gradients of $492 \mathrm{G} / \mathrm{cm}$ and $118 \mathrm{G} / \mathrm{cm}$, respectively. Each has a length 
of $1.62 \mathrm{~cm}$ and they are separated by a drift distance of $0.49 \mathrm{~cm}$. The beam at the end of the second quadrupole is nearly paraxial in both planes and the ellipticity $\left(Y_{f} / X_{f}\right)$ is 108 . This extremely elliptic example may be of limited value for research purposes since the large aspect ratio would make spatial resolution in the thin dimension very challenging. Assuming a incident beam of radius $R_{b 0}=0.1 \mathrm{~cm}$ (corresponding to an existing commercial gun: Litton; model M707) the resulting beam would be an ellipse with major radius $1.57 \mathrm{~cm}$ and a minor radius of $0.014 \mathrm{~cm}$. However, this example does serve to illustrate several important features. A comparison in Fig. 2 of the beam trajectories with space-charge to the case where space-charge is neglected shows dramatic differences, even within the first quadrupole, demonstrating that it is a very important effect in beams with either large current density or low longitudinal velocity. In the drift region, space-charge affects the focused dimension strongly and bends the trajectory back to near-paraxial flow. In the absence of space-charge the focused ray ends up crossing the axis, and, after further defocusing by the second quadrupole, a magnified negative beam image is formed in that plane.

Due to the large changes in transverse position in both planes, there exists a near decoupling of force effects at the second quadrupole. Since the magnetic field scales with displacement, the second quadrupole bends the defocused ray back into paraxiality but has very little effect on the focused ray. Hence, in very elliptic cases only the first quadrupole gradient, drift length and the space-charge density strongly affect the trajectory of the focused ray. For a beam of a given energy and current density, changes in beam ellipticity while maintaining paraxial flow, may be made through careful adjustment of the quadrupole gradients and separation.

\section{A. Physical Quadrupole Magnetic Fields}

So far the quadrupole has been assumed to be ideal. The quadrupole magnetic field abruptly rises from zero, is constant over a length, $L$, and then abruptly returns to zero. Provided this length is recognized as an effective length of the physical quadrupole, this model is relatively useful for understanding the physics of the focusing. However, in an experimental design one has to consider realistic profiles where the effect of the fringe fields is modeled more carefully. After Septier [9], we choose a bell-shaped field model as shown in Fig. 3. Other field models representing experimentally measured profiles may be easily substituted. The bell-shaped model has a length of constant field from $-z_{0}$ to $z_{0}$ with a Lorenztian fringing field on either side. The function describing this field distribution is given by

$$
f(z)=\left\{\begin{array}{ccc}
\frac{1}{\left(1+\left(\frac{z+z_{0}}{b}\right)^{2}\right)^{2}} & \text { for } \quad-\infty<z<-z_{0} \\
\frac{1}{\left(1+\left(\frac{z-z_{0}}{b}\right)^{2}\right)^{2}} & \text { for } \quad-z_{0} \leq z \leq z_{0}<z<\infty
\end{array}\right.
$$

The constants $z_{0}$ and $b$ prescribe the width of the flat-top and rate of fall-off of the profile, and, in general, will be determined from an experimentally measured magnetic profile.

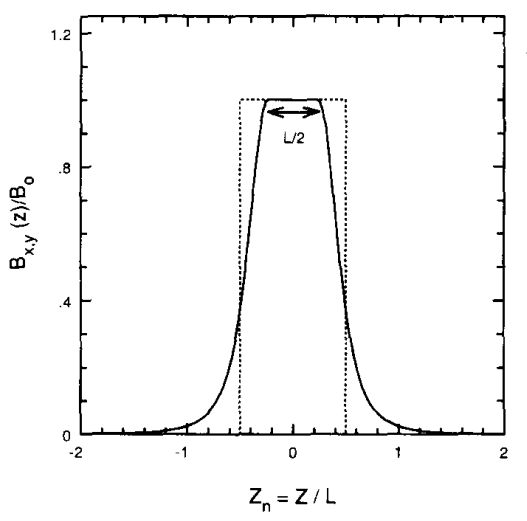

Fig. 3. The bell-shaped quadrupole model from (15). The dashed line is the equivalent ideal quadrupole model for $z_{0}=L / 4$.

For the purposes of this paper, we choose $2 z_{0}=L / 2$ and $b$ is found by constraining equal areas under the curve described by (15) with the ideal field profile of length $L$. This condition gives $b=L / \pi$. The two field profiles are compared in Fig. 3.

In the flat-top region the fields remain the ideal linear fields treated earlier. However, in the fringe region higherorder fields may be necessary in the field expansion. If the condition $X, Y \ll R_{0}$ is strictly satisfied throughout the particle trajectory, then the first-order fields given by (2), modulated by the profile function in (15) are sufficiently accurate. For highly elliptic flow this condition may not be completely satisfied and higher-order field terms must be included. To third order these fields are given by [9]

$$
\begin{aligned}
& B_{x}=-G f(z) y+\frac{G}{12} f^{\prime \prime}(z)\left(3 x^{2} y+y^{3}\right) \\
& B_{y}=-G f(z) x+\frac{G}{12} f^{\prime \prime}(z)\left(3 y^{2} x+x^{3}\right) \\
& B_{z}=-G f^{\prime}(z) x y
\end{aligned}
$$

where $G=B_{0} / R_{0}$ is the quadrupole gradient discussed previously. It is straightforward to show that these fields satisfy $\nabla \cdot \mathbf{B}=0$. Note that $B_{z}$ is considered third-order since in the equations of motion $B_{z}$ is cross-multiplied with $x^{\prime}$ or $y^{\prime}$.

We also allow for overlap and cancellation in the magnetic field profiles of the two quadrupoles and the drift region between the quadrupoles is no longer assumed to be field-free. If the quadrupoles are sufficently close, significant field cancellation can occur. In addition, we assume that magnetic shielding would be necessary to limit stray flux at the ends of the quadrupole system from affecting other components, such as the electron gun and the sheet-beam focusing system. As a reasonable assumption, we assume that shielding can be designed and placed such that the field profile given in (15) abruptly rises from zero at a position $L / 2$ from the center of the first magnet. Similarly, on the output end of the pair, we assume that the field is shielded such that the profile falls back to zero at $L / 2$ from the center of the second magnet. For the field profile considered here, the field amplitude is only about $40 \%$ of the 
peak, and the magnetic effects of the tail beyond this point are slight. The model considered here is easily changed to conform to any particular experimentally measured quadrupole system profile.

\section{B. Variations in Longitudinal Velocity}

We investigate the effect of longitudinal velocity variation for the case where $X^{\prime}, Y^{\prime} \ll 1$ may no longer be satisfied, but $X^{\prime 2}, Y^{\prime 2}$ are small enough that fourth-order terms in transverse velocity may be neglected. To reasonable approximation we may neglect space-charge depression and write $v_{z 0}^{2} \approx v_{x}^{2}+$ $v_{y}{ }^{2}+v_{z}{ }^{2}$. Using $\frac{d}{d t}=v_{z} \frac{d}{d z}$ we have

$$
v_{z}(z) \approx \frac{v_{z 0}}{\left(1+X^{\prime 2}+Y^{\prime 2}\right)^{\frac{1}{2}}}
$$

Noting that $\frac{d^{2} X}{d t^{2}}=v_{z}^{2} \frac{d^{2} X}{d z^{2}}+v_{z} \frac{d X}{d z} \frac{d v_{z}}{d z}$, we have for the $X$ equation of motion:

$$
\begin{aligned}
X^{\prime \prime}= & \left(\frac{v_{z}}{v_{z 0}}\right)^{2}\left(X^{\prime} X^{\prime \prime(1)}+Y^{\prime} Y^{\prime \prime(1)}\right) X^{\prime} \\
& +\left(\frac{\omega_{p 0} R_{b 0}}{v_{z}}\right)^{2} \frac{1}{X+Y} \\
& -\frac{e}{m v_{z}}\left(\frac{d \mathbf{r}}{d z} \times \mathbf{B}\right)_{x}+\frac{\epsilon_{n x}^{2}}{X^{3}}
\end{aligned}
$$

To simplify (18) we note that $X^{\prime \prime}$ (or $Y^{\prime \prime}$ ) appear on both sides of the equation. Hence, the procedure is to factor and substitute so that $X^{\prime \prime}$ (or $Y^{\prime \prime}$ ) only appears on the left-hand side, and then expand the denominators containing the small terms $X^{\prime}$ and $Y^{\prime}$. In the course of these calculations, use will be made of the expansions

$$
\begin{aligned}
\frac{1}{v_{z}} & \approx \frac{1}{v_{z 0}}\left[1+\frac{1}{2}\left(X^{\prime 2}+Y^{\prime 2}\right)\right] \\
\frac{1}{v_{z}^{2}} & \approx \frac{1}{v_{z 0}^{2}}\left[1+\left(X^{\prime 2}+Y^{\prime 2}\right)\right] .
\end{aligned}
$$

Substituting these expressions in (18), we proceed to expand and multiply, retaining only terms to third order in transverse velocity. As a short-cut, we note that the first term on the right of (18) already involves the second-order terms of $X^{\prime 2}$ and $X^{\prime} Y^{\prime}$. Hence, we use the superscript (1) on $X^{\prime \prime}$ and $Y^{\prime \prime}$ to denote that we need only consider the first-order components of these terms and the result will be fully third-order as intended. Assuming that the emittance term is small, we can, by inspection of (18), write down expressions for $X^{\prime \prime(1)}$ and $Y^{\prime \prime \prime}(1)$ that are accurate to first-order

$$
\begin{aligned}
& X^{\prime \prime(1)}=-\frac{e}{m v_{z 0}}\left(Y^{\prime} B_{z}-B_{y}\right)+\left(\frac{\omega_{p 0} R_{b 0}}{v_{z 0}}\right)^{2} \frac{1}{X+Y} \\
& Y^{\prime \prime(1)}=-\frac{e}{m v_{z 0}}\left(B_{x}-X^{\prime} B_{z}\right)+\left(\frac{\omega_{p 0} R_{b 0}}{v_{z 0}}\right)^{2} \frac{1}{X+Y} .
\end{aligned}
$$

Combining (16) and (18)-(22), normalizing as before and keeping only terms to third-order in transverse velocity, we obtain

$$
\begin{aligned}
X_{n}^{\prime \prime}= & -\left(k_{0} L\right)^{2}\left[1+\frac{1}{2}\left(\frac{R_{b 0}}{L}\right)^{2}\left(3 X_{n}{ }^{2}+Y_{n}{ }^{2}\right)\right] \\
& \times\left[f(z) X_{n}-\frac{R_{b 0}^{2}}{12} f^{\prime \prime}(z) X_{n}\left(3 Y_{n}{ }^{2}+X_{n}{ }^{2}\right)\right] \\
& +\frac{\left(k_{p} L\right)^{2}}{X_{n}+Y_{n}}\left[1+\left(\frac{R_{b 0}}{L}\right)^{2}\left(2 X_{n}{ }^{2}+Y_{n}{ }^{2}+X_{n}{ }^{\prime} Y_{n}{ }^{\prime}\right)\right] \\
& +\left(k_{0} L\right)^{2}\left(\frac{R_{b 0}}{L}\right)^{2} X_{n}{ }^{\prime} Y_{n}{ }^{\prime} \\
& \times\left[f(z) X_{n}-\frac{R_{b 0}^{2}}{12} f^{\prime \prime}(z) X_{n}\left(3 Y_{n}{ }^{2}+X_{n}{ }^{2}\right)\right] \\
& +\left(k_{0} L\right)^{2} \frac{R_{b 0}}{L} X_{n} Y_{n} Y_{n}{ }^{\prime} R_{b 0} f^{\prime}(z)+\frac{L^{2} \epsilon_{n x}^{2}}{R_{b 0}^{4} X_{n}{ }^{3}}
\end{aligned}
$$

The $Y_{n}$ ray equation is obtained from (23) by replacing $X_{n}$ with $Y_{n}$ and substituting $k_{0}{ }^{2} \rightarrow-k_{0}^{2}$ and $\epsilon_{n x} \rightarrow \epsilon_{n y}$. While not explicitly shown in (23), we note that the derivatives $f^{\prime}$ and $f^{\prime \prime}$ contain factors of $\frac{1}{L}$ and $\frac{1}{L^{2}}$ and (23) is fully normalized.

The normalization parameter $\frac{R_{b 0}}{L}$ is a measure of the strength of the higher-order field terms and the fringe field in the quadrupole. This is explicitly seen if we rewrite this parameter as $\frac{R_{b 0}}{L}=\frac{R_{b 0}}{R_{0}} \frac{R_{0}}{L}$. Thus, the effect of the fringe field is small for quadrupoles with a small ratio of radius-tolength. $\left(\frac{R_{0}}{L} \ll 1\right)$, provided that the transverse displacement of the envelope is small $\left(\frac{R_{b 0} X_{n}}{R_{0}}, \frac{R_{b 0} Y_{n}}{R_{0}} \ll 1\right)$. In highly elliptic beams, one must be careful that the quadrupole radius is sufficently large to justify the third-order magnetic field expansion in (16).

With the above discussion in mind, we further simplify (23) by dropping terms that scale as $\left(\frac{R_{b 0}}{L}\right)^{4}$. The equation of motion for $X_{n}$ becomes

$$
\begin{aligned}
X_{n}{ }^{\prime \prime}= & -\left(k_{0} L\right)^{2}\left[1+\frac{1}{2}\left(\frac{R_{b 0}}{L}\right)^{2}\left(3 X_{n}{ }^{2}+Y_{n}{ }^{2}\right)\right] f(z) X_{n} \\
& +\frac{1}{12}\left(k_{0} L\right)^{2} f^{\prime \prime}(z) X_{n} R_{b 0}{ }^{2}\left(3 Y_{n}{ }^{2}+X_{n}{ }^{2}\right) \\
& +\frac{\left(k_{p} L\right)^{2}}{X_{n}+Y_{n}}\left[1+\left(\frac{R_{b 0}}{L}\right)^{2}\left(2{X_{n}^{\prime}}^{2}+{Y_{n}^{\prime}}^{2}+X_{n}^{\prime} Y_{n}^{\prime}\right)\right] \\
& +\left(k_{0} L\right)^{2}\left(\frac{R_{b 0}}{L}\right)^{2} X_{n}^{\prime} Y_{n}^{\prime} f(z) X_{n} \\
& +\left(k_{0} L\right)^{2} \frac{R_{b 0}^{2}}{L} X_{n} Y_{n} Y_{n}{ }^{\prime} f^{\prime}(z)+\frac{L^{2} \epsilon_{n x}^{2}}{R_{b 0}^{4} X_{n}{ }^{3}}
\end{aligned}
$$

This equation (and the associated $Y_{n}$ equation) describe, to third-order, how the envelope of the beam evolves through the quadrupole lens system. Information about distortions (aberrations) in the beam cross-section can be gained from the solution of (24) for those envelope components which are not in the $x z$ or $y z$ planes. An experimental study of quadrupole aberrations [9] showed small distortions in the beam shape which, in principle, can be corrected in a careful lens system design. For the present purposes of this paper we examine 
only the envelope radius in the focusing plane $(x z)$ and the defocusing plane $(y z)$. Coupling terms for the ellipse elements in the these planes are zero except for in the denominator of the space-charge terms, and the ray equations are considerably simplified to give

$$
\begin{aligned}
X_{n}{ }^{\prime \prime}= & -\left(k_{0} L\right)^{2}\left[1+\frac{3}{2}\left(\frac{R_{b 0}}{L}\right)^{2} X_{n}{ }^{2}\right] f(z) X_{n} \\
& +\left(k_{0} L\right)^{2} \frac{R_{b 0}^{2}}{12} f^{\prime \prime}(z) X_{n}{ }^{3}+\frac{\left(k_{p} L\right)^{2}}{X_{n}+Y_{n}} \\
& \times\left[1+2\left(\frac{R_{b 0}}{L}\right)^{2} X_{n}{ }^{2}\right]+\frac{L^{2} \epsilon_{n x}^{2}}{R_{b 0}^{4} X_{n}^{3}} \\
Y_{n}^{\prime \prime}= & +\left(k_{0} L\right)^{2}\left[1+\frac{3}{2}\left(\frac{R_{b 0}}{L}\right)^{2} Y_{n}{ }^{2}\right] f(z) Y_{n} \\
& -\left(k_{0} L\right)^{2} \frac{R_{b 0}^{2}}{12} f^{\prime \prime}(z) Y_{n}{ }^{3}+\frac{\left(k_{p} L\right)^{2}}{X_{n}+Y_{n}} \\
& \times\left[1+2\left(\frac{R_{b 0}}{L}\right)^{2} Y_{n}{ }^{2}\right]+\frac{L^{2} \epsilon_{n y}^{2}}{R_{b 0}^{4} Y_{n}^{3}} .
\end{aligned}
$$

Finally, it is acknowledged that the emittance term was handled in an approximate manner in the above analysis. Its inclusion illustrates that the effect of nonzero emittance on the beam envelope is not negligible, and should be included in a careful design. Strictly speaking, however, there is difficulty in relating the emittance as defined above with an experimentallymeasurable rms emittance. Several authors [7], [10] have derived envelope equations for the effective beam width and emittance which are easily related to measurable parameters in the laboratory. The derivations of these rms envelope equations require linear focusing forces (and elliptical beam symmetry). Hence, the rms equations would not rigorously apply in the case where higher-order field and velocity variation effects are included in the ray equations. However, in the spirit of the small-term corrections applied in the above equations, an rms approach may only represent a reasonable error in the analysis.

\section{Elliptical Sheet Beam Design Case}

In order to illustrate the feasibility of quadrupole formation of elliptical sheet beams, we give a design example using an existing commercial round-beam electron gun (Litton; M690). Equations (25) and (26) were solved numerically for a $10 \mathrm{keV}$ beam with a current density of $2 \mathrm{~A} / \mathrm{cm}^{2}$. The resulting beam envelope in the focusing and defocusing planes is shown in Fig. 4. Assuming a beam radius of $0.6 \mathrm{~cm}$ at the quadrupole entrance, the output beam has a major radius of $3.61 \mathrm{~cm}$, minor radius of $0.16 \mathrm{~cm}$ and an ellipticity of 22.5 . The first quadrupole has a gradient of $64 \mathrm{G} / \mathrm{cm}$ and the second has a gradient of $18 \mathrm{G} / \mathrm{cm}$. A plot of the normalized magnetic field profile used in this simulation is shown in Fig. 5. The magnets have a flat-top region of length $1.41 \mathrm{~cm}$, and the separation between the centers of the the quadrupoles is $3.1 \mathrm{~cm}$. Assuming a quadrupole radius of $6 \mathrm{~cm}$, the magnetic fields at the pole tips for the two quadrupoles are a modest $383 \mathrm{G}$ and $106 \mathrm{G}$.

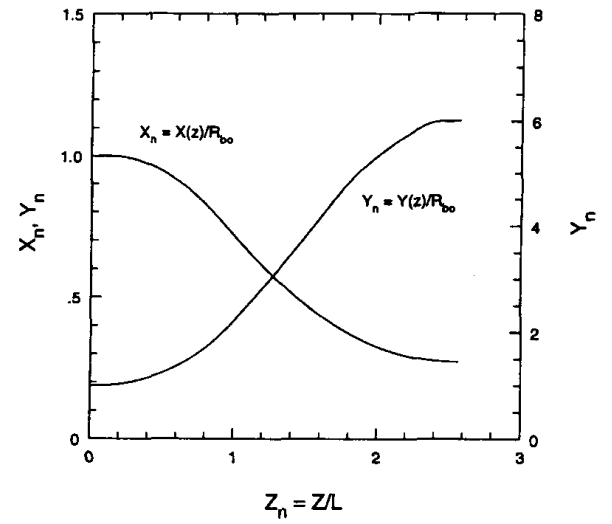

Fig. 4. Envelope trajectories for a sheet-beam with ellipticity of 22.5 using round-beam parameters from an existing commercial gun. Third-order effects of longitudinal velocity variations and a realistic magnetic field are included in the model.

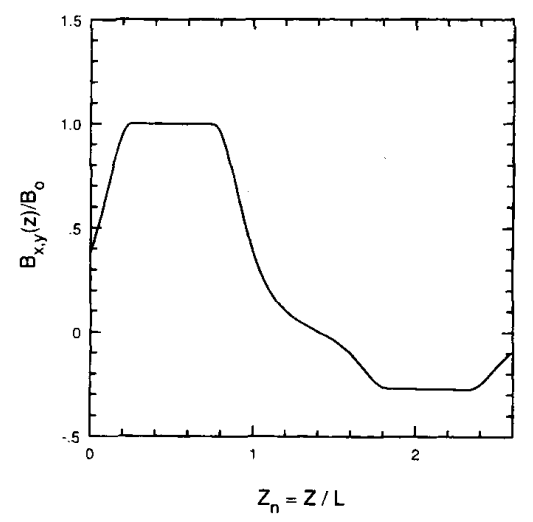

Fig. 5. Magnetic field used in calculating the envelope of Fig. 4. Only modest quadrupole gradients of $64 \mathrm{G} / \mathrm{cm}$ and $18 \mathrm{G} / \mathrm{cm}$ are required.

\section{Design CONSIDERATIONS AND SUMmary}

Several design issues of an elliptical beam forming system must also be considered. If the input beam is generated by a flux-free Pierce diode, then magnetic shielding must be designed such that the quadrupole field begins sharply at the electrostatic focus of the beam. For an experimental program it may be desirable to assemble or disassemble the gun from the vacuum tube and a quadrupole system design may require a drift space that can accomodate a vacuum valve. Additionally, the sheet-beam focusing configuration (PCM or wiggler) must be placed in a position to take advantage of the paraxial beam at the exit of the quadrupole system. It may be necessary to overfocus the beam slightly at the exit in order for the sheetbeam periodic focusing system to be effective at the matched position.

Aberations due to higher-order magnetic field components should be studied to determine their effect on the entire beam envelope. Such higher order effects may distort the beam cross-section from being purely elliptical and could cause problems within the sheet-beam focusing system. An interesting experimental study of quadrupole focusing aberrations was 
conducted by Septier et. al. [9] using a hollow beam. It was found that, for a carefully designed quadrupole, the effect of aberrations on the beam cross-section was modest. However, it must be kept in mind that the use of a hollow beam minimizes the effects of space-charge and beam emittance on the profile. Further studies will use a three-dimensional particle trajectory code to examine the range of validity of the model presented in this paper and to investigate the effects of field aberrations.

Magnetic quadrupole lens systems appear to be an attractive option for forming elliptical sheet electron beams. This method is particularly suited for research, since it can utilize existing round-beam sources and changes in beam ellipticity can be made without breaking the vacuum envelope. Beam transport through quadrupoles is reasonably well understood and the theory is relatively straightforward. Quadrupole formation systems coupled to round-beam sources could prove more feasible to design and build than a sheet-beam electron gun. In the design example presented here, beam parameters from an existing electron gun $\left(2 \mathrm{~A} / \mathrm{cm}^{2}, 10 \mathrm{keV}, R_{b 0}=0.6 \mathrm{~cm}\right)$ were used and the solution to the envelope equations indicated that a paraxial sheet-beam with a major radius of $3.61 \mathrm{~cm}$, minor radius of $0.16 \mathrm{~cm}$ and ellipticity of 22.5 is possible.

\section{ACKNOWLEDGMENT}

The authors would like to acknowledge helpful discussions with Dr. B. McVey, Dr. R. True, Dr. G. Schietrum, and Dr. J. Scharer.

\section{REFERENCES}

[1] B. D. McVey, M. A. Basten, J. H. Booske, J. Joe, and J. E. Scharer, "Analysis of rectangular waveguide-gratings for amplifier applications," IEEE Trans. Microwave Theory Tech., to be published.

[2] Z. X. Zhang, V. L. Granatstein, W. W. Destler, S. W. Bidwell, J. Rodgers, S. Cheng, T. M. Antonsen, B. Levush, and D. J. Radack, "Experimental and numerical studies of sheet electron beam propagation through a planar wiggler magnet," IEEE Trans. Plasma Sci., vol. 21 pp. 760-767, Dec. 1993

[3] T. M. Antonsen and E. Ott, "Velocity shear driven instabilities of an unneutralized electron beam," Phys. Fluids, vol. 18, pp. 1197-1208, 1975.

[4] J. H. Booske, B. D. McVey, and T. M. Antonsen, "Stability and confinement of nonrelativistic sheet electron beams with periodic cusped magnetic focusing," J. Appl. Phys., vol. 73, no. 9, pp. 4140-4155, 1993.

[5] P. Grivet, Electron Optics. Oxford, U.K.: Pergamon, 1972.
[6] E. Regenstreif, "Focusing with quadrupoles, doublets, and triplets," Focusing of Charged Particles, vol. 1, pp. 353-410, 1967.

[7] P. M. Lapostolle, "Possible emittance increase through filamentation due to space charge in continuous beams," IEEE Trans. Nuc. Sci., vol. 18, 1971.

[8] J. D. Lawson, The Physics of Charged-Particle Beams . Oxford, U.K.: Oxford Univ. Press, 1977.

[9] A. Septier, "Strong-focusing lenses," Electronics and Electron Physics, vol. 14, pp. 86-203, 1961.

[10] F. J. Sacherer, "RMS envelope equations with space charge," IEEE Trans. Nuc. Sci., vol. 18, 1971.

Mark A. Basten was born in Iowa City, IA, on June 14, 1966. He received a B.S. in physics from the University of Iowa in 1988. He was awarded a DOE Magnetic Fusion Science Fellowship and attended MIT, where he worked on the development of high-power millimeter-wave sources. Topics of research included the experimental investigation of a $130-145 \mathrm{GHz}$ gyrotron-BWO, the theoretical study of a $95 \mathrm{GHz}$ gyrotron-TWT design and efficiency studies on highly-overmoded gyrotron oscillators. After receiving a M.S. in physics he attended the University of Wisconsin-Madison, where he is working toward a Ph.D. in electrical engineering.

His current research interests include Cerenkov amplifiers and the stable formation and propagation of sheet electron beams.

Mr. Basten is a member of Sigma Pi Sigma and Phi Beta Kappa.

John H. Booske (S'82-M'85-SM'93) recieved the Ph.D. degree in nuclear engineering from the University of Michigan, Ann Arbor, in 1985. Topics of graduate research included high-power $\mathrm{CO}_{2}$ lasers and electron-cyclotron resonant heating and spontaneous cyclotron emissions from magnetically confined hot-electron plasmas.

In 1985 he began work in the Laboratory for Plasma Research, University of Maryland-College Park, as a Research Associate, studying hot ion microinstabilities in magnetic mirror plasmas. He subsequently joined the Research and Graduate faculties at the University of Maryland, with responsibility for research in millimeter-wave free electron lasers. He is currently a faculty member in the Department of Electrical and Computer Engineering, University of Wisconsin-Madison. His research interests include electron beam and plasma radiation sources, plasma heating, multimoded electromagnetic cavity phenonmena, plasma modification of materials' surfaces, and the interactions of high-power microwaves with solid state materials.

James P. Anderson was born in Madison, WI, on June 10, 1972. He is working towards his B.S. degree in electrical engineering from the University of Wisconsin at Madison.

$\mathrm{He}$ is currently doing research for the Department of Electrical and Computer Engineering. 